\section{Versatile derivatives of carbohydrate-binding modules for imaging of complex carbohydrates approaching the molecular level of resolution}

\author{
Shi-You Ding1, Qi Xu1, Mursheda K. Ali¹, John O. Baker1, Edward A. Bayer², \\ Yoav Barak2, Raphael Lamed ${ }^{3}$, Junji Sugiyama ${ }^{4}$, Garry Rumbles ${ }^{1}$, and \\ Michael E. Himmel ${ }^{1}$ \\ ${ }^{1}$ National Renewable Energy Laboratory, Golden, CO, USA, ${ }^{2}$ Weizmann Institute of \\ Science, Rehovot, Israel, ${ }^{3}$ Tel Aviv University, Tel Aviv, Israel, and ${ }^{4}$ Kyoto University, \\ Kyoto, Japan
}

BioTechniques 41:435-443 (October 2006)

doi 10.2144/000112244

The innate binding specificity of different carbohydrate-binding modules (CBMs) offers a versatile approach for mapping the chemistry and structure of surfaces that contain complex carbohydrates. We have employed the distinct recognition properties of a double His-tagged recombinant CBM tagged with semiconductor quantum dots for direct imaging of crystalline cellulose at the molecular level of resolution, using transmission and scanning transmission electron microscopy. In addition, three different types of CBMs from families 3, 6, and 20 that exhibit different carbohydrate specificities were each fused with either green fluorescent protein (GFP) or red fluorescent protein (RFP) and employed for double-labeling fluorescence microscopy studies of primary cell walls and various mixtures of complex carbohydrate target molecules. CBM probes can be used for characterizing both native complex carbohydrates and engineered biomaterials.

\section{INTRODUCTION}

Carbohydrates make up a vital and ubiquitous class of essential nutrients and play important roles in innumerable biological processes, including cell recognition, intercellular signal transduction, cell growth, and cell differentiation (1-3). However, progress toward understanding their structure and function lags behind other biomolecules, such as nucleic acids and proteins. This is primarily due to the high degree of complexity and heterogeneity displayed by carbohydratecontaining materials. Today, methods available for characterizing complex carbohydrates include spectroscopy [mass spectroscopy (MS), Fourier transform infrared, Raman, and nuclear magnetic resonance (NMR) spectroscopies], immunochemical labeling, electron microscopy, and X-ray crystallography. These approaches often have low spatial resolution (micron level or above) or require extensive sample preparation that may alter the molecular structure of the polysaccharide sample.
The plant cell wall is an intricate conglomerate that includes numerous polysaccharides as major components (e.g., cellulose, xyloglucan, xylans, mannans, arabinans, and pectins). The synthesis and incorporation of the various wall polysaccharides into a durable fibrous network has been the subject of numerous recent investigations (4). For this purpose, various fluorescence and electron microscopic labeling methods have proved especially effective. In this context, immunocytochemical (5-8), enzyme(9-14), and lectin-based $(9,15)$ labeling techniques have been developed to examine the in situ distribution of plant cell wall polysaccharides.

Carbohydrate-binding modules (CBMs) are noncatalytic protein modules found in many carbohydratehydrolyzing enzymes, such as the cellulases and hemicellulases. They function as recognition modules that convey the catalytic modules of these enzymes to the target substrate. Several hundred CBMs have been identified to date, and these have been grouped into
45 families using amino acid sequence similarity algorithms (afmb.cnrs-mrs. fr/CAZY/index.html). The structures and ligand specificities of many CBMs have also been determined experimentally (16). Three types of CBMs have now been proposed based on their structure and function; these include type-A, which bind to specific surfaces of insoluble, crystalline cellulose and/or chitin; type-B, which bind to individual glycan chains; and type-C, or small sugar-binding CBMs, which have lectin-like properties and bind to mono-, di-, or trisaccharides.

The fine-tuned polysaccharide recognition capacity of the CBMs has prompted their development as precise molecular probes for mapping the chemistry and structure of carbohydratecontaining materials (16-19). CBMs have thus been used for fluorescence labeling of plant cell walls (20-22) and for ultrastructural labeling of Valonia cellulose crystals (23). Notably, following the original submission of this paper, differential recognition of plant cell walls in different tissues by different xylan-specific CBMs was reported (22). However, these published descriptions of CBM probes require complex labeling procedures or the development of monoclonal antibodies. In addition, imaging at the nanometer scale with high resolution has not been described.

In the present study, we describe an approach that simplifies the labeling procedures, requires minimal sample preparation, and heightens substantially the resolution power. Three CBMs from three different families that possess three distinctive polysaccharide specificities were engineered with dual His-tags to facilitate their coordination to highly luminescent quantum dots for correlative imaging using fluorescence and electron microscopy. In addition, the CBMs were each expressed as fusion proteins, with either green fluorescent protein (GFP) or redfluorescent protein (RFP), to allow direct imaging using fluorescence microscopy at the near-molecular level of resolution. This approach complements newly developed techniques for visualizing the interaction of $\mathrm{CBMs}$ with carbohydrate composites and is potentially capable of single molecule detection. 


\section{MATERIALS AND METHODS}

\section{Sample Preparation}

Crystalline cellulose was prepared from the algae, Valonia ventricosa, as described previously $(24,25)$. $V$. ventricosa vesicles were harvested and rinsed with distilled water, then were boiled in $2 \% \mathrm{NaOH}$ for $6 \mathrm{~h}$ (changing fresh $2 \% \mathrm{NaOH}$ every $2 \mathrm{~h}$ ). The purified vesicles were neutralized with dilute $\mathrm{HCl}$ and washed with distilled water. For the preparation of cellulose crystals, purified vesicles were homogenized for $10 \mathrm{~min}$ at $10,000 \mathrm{rpm}$. The resulting suspensions were hydrolyzed by stirring for $5 \mathrm{~h}$ in $3.5 \mathrm{M} \mathrm{HCl}$ and then neutralized with a few drops of $\mathrm{NaOH}$ solution and washed with distilled water. The suspensions of Valonia cellulose crystal were stored at $4^{\circ} \mathrm{C}$ in distilled water containing $0.01 \%$ $(\mathrm{w} / \mathrm{v}) \mathrm{NaN}_{3}$. Small aliquots of cellulose crystal stock suspension were diluted in $20 \mathrm{mM}$ Tris buffer, $\mathrm{pH} 8.0$, to make the final concentration of $1 \mathrm{mg} / \mathrm{mL}$ used for this study.

Phosphoric-acid-treated cellulose was produced by preparing a cellulose slurry $(1 \mathrm{mg} / \mathrm{mL})$ in dilute $(2 \% \mathrm{v} / \mathrm{v})$ phosphoric acid overnight at room temperature, followed by extensive washing with distilled water to neutral $\mathrm{pH}$.

Xyloglucan-bound cellulose was prepared by adding $30 \mu \mathrm{L}$ xyloglucan solution $(10 \mathrm{mg} / \mathrm{mL})$ to a $50-\mu \mathrm{L}$ cellulose suspension, followed by vortex mixing, centrifugation at $4000 \times$ $g$ for $1 \mathrm{~min}$, and washing steps. This substrate was finally suspended in 50 $\mu \mathrm{L}$ Tris buffer.

Starch granules were purchased from Sigma-Aldrich (St. Louis, MO, USA). The granules were suspended in distilled water $(10 \mathrm{mg} / \mathrm{mL})$, and $0.5 \mathrm{~mL}$ was mixed with an equivalent suspension of Valonia cellulose (1 $\mathrm{mg} / \mathrm{mL}$ ). The mixture was washed with distilled water three times by centrifugation $(4000 \times g$ for $1 \mathrm{~min})$ and suspended in $1 \mathrm{~mL} 20 \mathrm{mM}$ Tris buffer.

The glycoprotein, cellobiohydrolase (Cel7A), was purified from the fermentation broth of induced Trichoderma reesei (26). Cel7A is a naturally glycosylated protein, which contains a catalytic and a family-1 CBM that mediates attachment to the cellulose substrate. Thirty microliters Cel7A $(1 \mathrm{mg} / \mathrm{mL}$ in $50 \mathrm{mM}$ acetate buffer, $\mathrm{pH} 5.0$ ) were added to $50 \mu \mathrm{L}$ $1 \mathrm{mg} / \mathrm{mL}$ cellulose suspension, gently mixed for $30 \mathrm{~min}$, followed by centrifugation ( $4000 \times g$ for $1 \mathrm{~min}$ ) and three additional washes with acetate buffer. The final pellet was suspended in 20 $\mu \mathrm{L}$ acetate buffer.

Maize (Zea mays L.) was grown in the lab greenhouse. Parenchyma tissue was carefully separated from stem pith of a mature plant and immediately pulverized in liquid nitrogen. Small pieces of primary cell walls were washed and finally suspended in distilled water.

\section{Sample Labeling}

CBMs were cloned, expressed, and purified (see the supplementary material available online at www. BioTechniques.com). CBM labeling was performed in 1.5-mL Eppendorf ${ }^{\circledR}$ tubes, with $50 \mu \mathrm{L}$ sample (suspension of Valonia cellulose or amorphous cellulose, starch/cellulose mixture, xyloglucan-bound-cellulose, or Cel7Abound-cellulose, prepared above) and $20 \mu \mathrm{L}$ desired CBM solution (1 $\mathrm{mg} / \mathrm{mL})$ in $20 \mathrm{mM}$ Tris buffer $(\mathrm{pH}$ 8.0). The sample was brought to a total slurry volume of $100 \mu \mathrm{L}$ with Tris buffer, mixed gently for $30 \mathrm{~min}$, and washed three times by centrifugation (4000× $g$ for $1 \mathrm{~min}$ ) using Tris buffer. The final pellet was suspended in 20 $\mu \mathrm{L} 20 \mathrm{mM}$ Tris buffer. These samples were used for fluorescence microscopy measurement.

\section{Colloidal Quantum Dots}

$(\mathrm{CdSe}) \mathrm{ZnS}$ core-shell quantum dots (QDs) were prepared as described previously (27). The surface capping groups were exchanged for mercaptopropionic acid (MPA) to confer solubility in $20 \mathrm{mM}$ Tris buffer, $\mathrm{pH}$ 6.5 .

The CBM-HIS probe was first bound to cellulose crystals as described above. Ten microliters QDs solution were then added to the CBM-HISbound cellulose, followed by three centrifugation (at $4000 \times g$ for $1 \mathrm{~min}$ )/ wash steps. The QD-labeled, CBMbound cellulose was then suspended in $20 \mu \mathrm{L}$ Tris buffer.

\section{Atomic Force Microscopy}

Five microliters Valonia cellulose suspension $(1 \mathrm{mg} / \mathrm{mL}$ in water) were spin-coated on a fresh-cut mica, which was precoated by polyhistidine $(1 \mathrm{mg} /$ $\mathrm{mL}$ in water). Atomic force microscopy (AFM) [MultiMode ${ }^{\mathrm{TM}}$ Scanning Probe Microscope (SPM); Veeco Instruments, Woodbury, NY, USA] was operated in tapping mode underwater using a flow cell.

\section{Fluorescence Microscopy}

One microliter freshly prepared sample was placed between two glass coverslips. Direct imaging was performed by fluorescence microscopy using an Olympus IX71 inverted microscope (Olympus America, Center Valley, PA, USA), equipped with a DP70 charge-coupled device (CCD) camera, mercury lamp as excitation source, fluorescence cubes including U-MNU $(\mathrm{Ex}=360-370 \mathrm{~nm}$ and $\mathrm{Em} \geq$ 420), U-MNB (Ex $=470-490 \mathrm{~nm}, \mathrm{BA}$ $=515 \mathrm{~nm}$, and $\mathrm{DM}=500)$, U-MNG $(\mathrm{Ex}=530-550 \mathrm{~nm}$ and $\mathrm{Em} \geq 570$ $\mathrm{nm})$, objective lens $(150 \times$ Uapro, oil immersion, and N.A. = 1.45).

\section{Electron Microscopy}

Freeze-dried samples were prepared by the wet-film method described in Reference 28. Samples were embedded in a very thin $(<1$ $\mu \mathrm{m})$ layer of water, deep-frozen, and slowly freeze-dried while warming from $-80^{\circ} \mathrm{C}$. Transmission electron microscopy (TEM) images were captured with a CCD camera using DigitalMicrograph ${ }^{\mathrm{TM}}$ software (Gatan, Pleasanton, CA, USA) on a Tecnai G2 20 microscope (FEI, Hillsboro, OR, USA) operating at $200 \mathrm{kV}$. Scanning transmission electron microscopy (STEM) was performed at $40 \mathrm{keV}$ with a cold field-emission gun, a 0.3$\mathrm{nm}$ probe, and two annular detectors subtending 15-40 and 40-200 mRadian 
at the specimen (STEM, Brookhaven National Laboratory STEM facility, Upton, NY, USA; www.biology.bnl. gov/stem/stem.html). A specimen changer air lock permitted vacuum transfer of frozen or freeze-dried specimens from a separate ion-pumped freeze dryer or between instruments.

\section{RESULTS AND DISCUSSION}

Three different CBMs (Table 1) were selected as examples in this study to demonstrate the variety in their binding specificities for different carbohydrate-containing materials and their application as selective labeling agents for high-resolution microscopy. $\mathrm{CtCBM} 3$ was originally cloned from a Clostridium thermocellum CipA protein and has been reported to be a type A surface-binding CBM with high affinity for crystalline cellulose $(29,30)$. SlCBM20 is from a Streptomyces lividans $\alpha$-amylase (AmlA) and is a putative chain-binding CBM that recognizes starch. $C t$ CBM6 is cloned from a $C$. thermocellum xylanase (XynZ). Based on experimental studies and Xray structures (31-33), members from this particular family of CBMs exhibit two potential binding sites with distinct ligand recognition for glucan and xylan oligosaccharides. Indeed, the $C t \mathrm{CBM} 6$ used for these studies is highly similar in sequence and conserved binding residues to the well-studied Xyn11A CBM6 from the same bacterium. The Xyn11A CBM6 was previously demonstrated to bind primarily to xylan, but also interacts weakly with $\beta$-glucan, soluble forms of cellulose, cellohexaose, and xylooligosaccharides (31). The XynZ CBM6 exhibits similar preference for xylan but also binds to xyloglucan and noncrystalline cellulose (Table 1).

Each CBM was engineered in three different recombinant forms with different functional tags: CBM-HIS (containing two $6 \times$-His-tags, at the $\mathrm{N}$ and $\mathrm{C}$ termini), CBM-GFP (peak emission $509 \mathrm{~nm}$ ), and CBM-RFP (peak emission $585 \mathrm{~nm}$ ). Six carbohydrate-containing materials, including crystalline and amorphous cellulose, starch, xyloglucan, glycoproteins, and plant primary cell walls, were used in this study for demonstrating the specific binding of the CBMs and their use in correlative labeling studies.

Cellulose is a linear polymer of $\mathrm{D}$ glucose linked by the $\beta-1,4$-glucosidic bond. The glucan chains pack side-byside to form an insoluble macromolecule - the microfibril—by a combined intra- and intermolecular hydrogen bonding network. Subtle changes in hydrogen bonding between adjacent

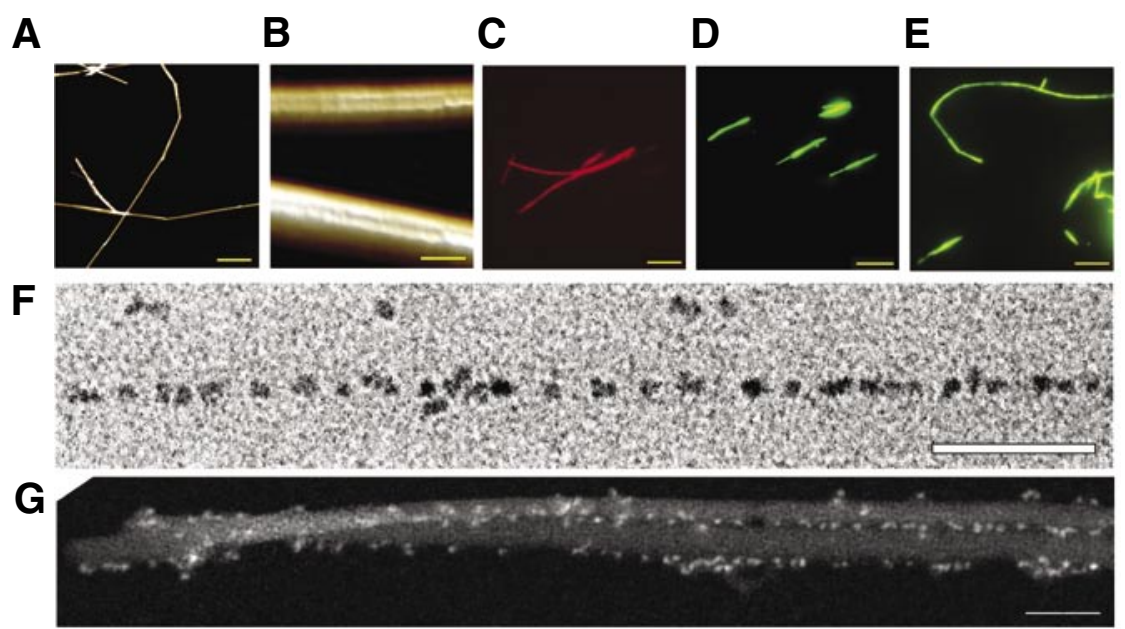

Figure 1. Micrographs of single Valonia cellulose crystals and CBM3 binding. Atomic force micrograph of cellulose crystals (panel A, scale bar $=500 \mathrm{~nm}$ ) and higher magnification of the hydrophobic face of a single crystal (panel B, scale bar $=50 \mathrm{~nm}$ ). Fluorescence microscope imaging of cellulose crystals labeled with (C) $C t$ CBM3-RFP, (D) $C t$ CBM3-GFP, and (E) $C t$ CBM3-HIS/QDs, scale bars, 5 $\mu \mathrm{m}$. (F) TEM and (G) STEM of cellulose crystal bound with CtCBM3-HIS/QDs. Quantum dots (QDs) are visible as dark spots in the TEM image, and white dots in dark-field STEM image. Scale bars, $50 \mathrm{~nm}$. TEM, transmission electron microscopy; STEM, scanning transmission electron microscopy.

polymer chains of native cellulose may generate crystalline, subcrystalline, and amorphous celluloses (34). From any given cellulose source, these regions are not particularly discrete, and there is a gradation among these fractions along the length of a single microfibril. Valonia cellulose is among the most crystalline of all celluloses. The Valonia cellulose $1 \alpha$ crystals selected for this study were about $20 \mathrm{~nm}$ in diameter and usually $10-15 \mu \mathrm{m}$ in length $(23,35)$, which was confirmed by AFM using a MultiMode SPM imaged in water (36) (Figure 1, A and B). The length of Valonia cellulose crystals varies within a given preparation, and the crystals sometimes associate to form bundles. The cellulose crystals exhibit distinct hydrophobic faces (the 1, 1, 0 or planar face) and two hydrophilic faces (the $1,0,0$ and $0,0,1$ or step faces) (24). Amorphous cellulose, in which portions of the cellulose crystal are disrupted, was produced by mixing Valonia cellulose with dilute $(2 \% \mathrm{v} / \mathrm{v})$ phosphoric acid. The resultant product is believed to emulate, to some extent, the amorphous regions dispersed in crystalline cellulose (37). The $C t \mathrm{CBM} 3$ GFP and $C t$ CBM3-RFP probes bind strongly to crystalline and phosphoric acid-treated cellulose, and the single cellulose crystal could be visualized using fluorescence microscopy (Figure 1, C-E). Unlike $C t \mathrm{CBM} 3$, the $C t \mathrm{CBM} 6$ probes demonstrated no detectable binding to crystalline Valonia cellulose. Upon correlative binding using the CtCBM3-RFP and CtCBM6-GFP probes however, we have confirmed that $C t$ CBM6-GFP binds to amorphous regions of phosphoric acid-treated cellulose (Figure 2A). To identify the preferred face of the cellulose crystal for $\mathrm{CtCBM} 3$ binding, $\mathrm{CtCBM} 3-\mathrm{HIS}$ was first introduced to the sample of Valonia cellulose, followed by the addition of semiconductor (CdSe) $\mathrm{ZnS}$ QDs. The highly luminescent colloidal QDs (peak emission at $571 \mathrm{~nm}$ ) were previously treated with mercaptopropionic acid to confer water solubility (27). In this way, we were able to label the bound $\mathrm{CtCBM} 3-\mathrm{HIS}$ using the strong coordinate covalent interaction between the His-containing peptides and the $\mathrm{ZnS}$ shell of the QDs (38). In this context, the His-tags at the $\mathrm{N}$ and $\mathrm{C}$ termini of 
the $\mathrm{CBH} 3$ molecule are positioned distally to the cellulose-binding surface (29), thus providing oriented labeling of the molecule by the QDs. The resulting QD-labeled sample was imaged using fluorescence microscopy (Figure 1E), TEM (Figure 1F), and STEM (Figure $1 \mathrm{G})$. The latter image clearly shows that $\mathrm{CtCBM} 3$-HIS binds to only one face of the cellulose crystal. This face is about $2 \mathrm{~nm}$ wide and was previously reported by Lehtio et al. to be the planar hydrophobic $(1,1,0)$ face $(23)$ as predicted earlier (29). The resolution afforded by this approach is far superior to indirect immunogold-based labeling, which was evaluated statistically in the previous study (23). The extensive labeling along the entire $(1,1,0)$ face of the crystal is unequivocal in Figure 1G (compared with figure 4 in Reference 23), requiring no additional mapping of the label, thus verifying the results of the Lehtio et al. and demonstrating the utility of the method described in this communication.

The versatility of employing different CBM-bearing GFP- and RFPfusion proteins for double-labeling fluorescence-microscopy studies was further demonstrated using mixtures of complex carbohydrate-containing materials. Native starch granules contain a mixture of amylose ( $\alpha-1,4-\mathrm{D}$-glucan) and amylopectin ( $\alpha-1,4-D$-glucan backbone with $\alpha-1,6$-glucose linked branch points approximately every 20 backbone residues). Correlative binding of $\mathrm{CtCBM} 3-\mathrm{GFP}$ and SlCBM20-RFP to a mixture of starch granules and crystalline cellulose showed distinct and unique recognition for these two CBMs. CtCBM3-GFP bound only to the cellulose crystals, whereas SlCBM20RFP bound only to starch (Figure 2B). These results demonstrate that the $\mathrm{CtCBM} 3$ and $S l C B M 20$ probes specifically label the $\beta$ - and $\alpha-1,4$-glycosidic linkages, respectively.

Tamarind xyloglucan (Megazyme, Wicklow, Ireland) used in this study is a branched polysaccharide, which has a molecular weight of $202 \mathrm{kDa}$, comprising Ara:Gal:Xyl:Glc at a ratio of 3:16:36:45. Soluble xyloglucan was layered on cellulose crystal surfaces by simple mixing, followed by extensive centrifugation and washing steps. Correlative binding of the

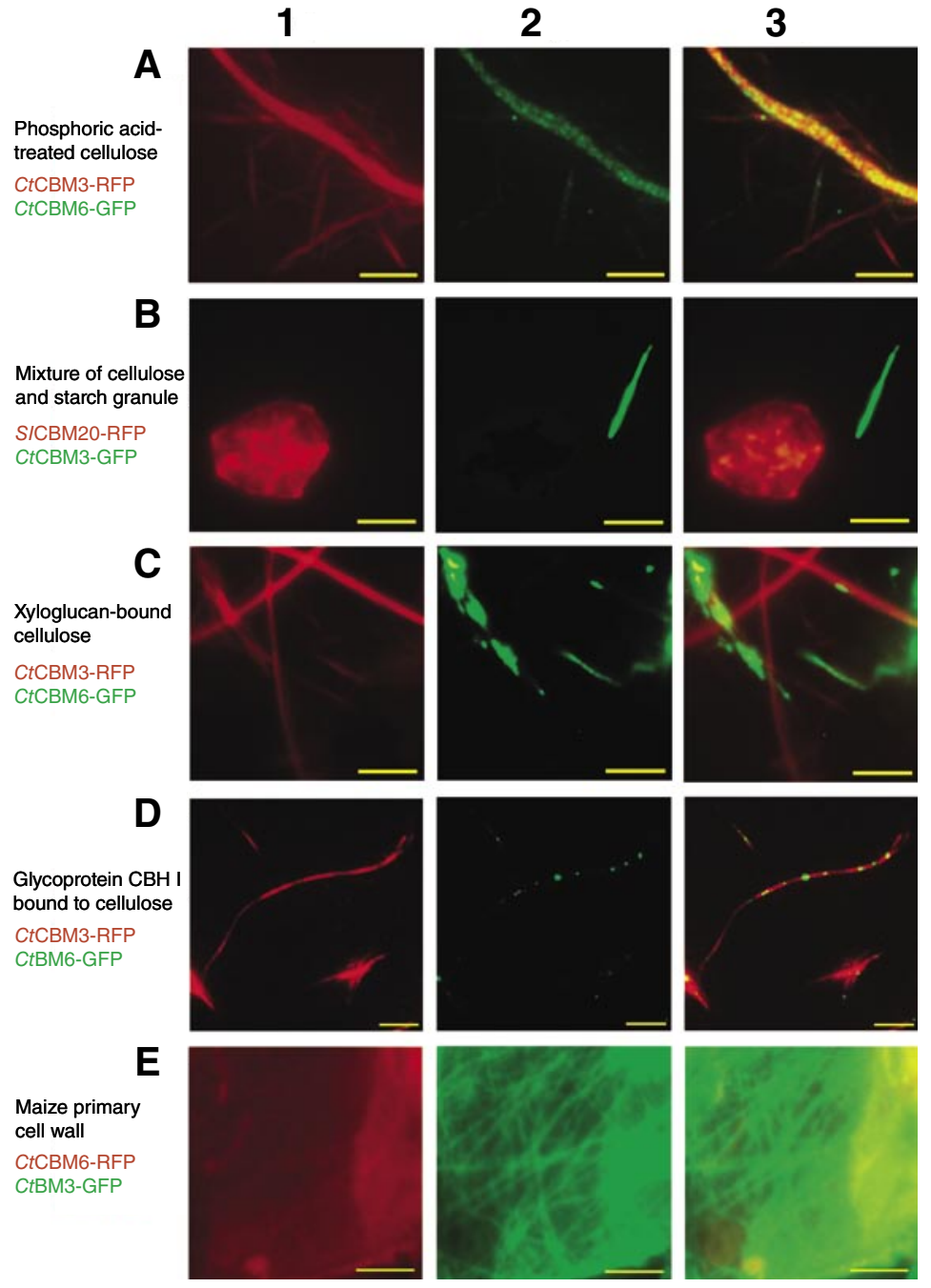

Figure 2. Fluorescence micrographs showing distinct recognition of correlative binding of two different carbohydrate-binding molecules (CBMs) to various carbohydrate-containing materials. Micrographs in column 1 show red fluorescent protein (RFP) labeling of the respective samples; those in column 2 show green fluorescent protein (GFP) labeling; and those in column 3 the two combined. (A) Phosphoric acid-treated preparations of Valonia cellulose, co-labeled with $\mathrm{CtCBM} 3-\mathrm{RFP}$ and $\mathrm{CtCBM6-}$ GFP. Note the noncrystalline patches in the cellulose fiber associated with the CtCBM6-based probe in (panel A, column 2) and (panel A, column 3). (B) Correlative binding of a mixture of Valonia cellulose and starch granules, labeled with SICBM20-RFP and CtCBM3-GFP. Note the explicit discrimination between the SlCBM20-mediated labeling of the starch granule and the $\mathrm{CtCBM}$-induced labeling of the cellulose crystal. (C) Xyloglucan-cellulose mixture, labeled as in panel A. (D) Selective labeling of a cellulose-bound glycoprotein (Trichoderma reesei Cel7A), labeled as in panel A. (E) Correlative mapping of the maize parenchyma cell wall. CtCBM3-GFP highlights the cellulose macrofibrils versus the CtCBM6-RFP that labels the primary cell wall matrices. Scale bars, $5 \mu \mathrm{m}$.

CtCBM3-RFP and CtCBM6-GFP probes to this substrate showed that CtCBM6-GFP bound only to the adsorbed xyloglucan, whereas the $C t$ CBM3-RFP selectively labeled the Valonia cellulose crystals in the sample (Figure 2C).

The glycoprotein cellobiohydrolase I (Cel7A) from $T$. reesei is a processive $\beta$-1,4-exo-glucanase that contains a glycoside hydrolase family-7 catalytic module, a family-1 CBM that binds to crystalline cellulose, and a heavily glycosylated linker peptide connecting the two. The CBM1 of $T$. reesei Cel7A has been reported to display high affinity for crystalline cellulose (39). Similar to $C t \mathrm{CBM} 3, \operatorname{Tr} \mathrm{CBM} 1$ binds to the narrow planar $(1,1,0)$ faces of the cellulose crystal (23). In the present study, Cel7A was first bound to Valonia cellulose crystals, followed by correlative binding 
Table 1. Carbohydrate-Binding Molecules and Their Ligands Used in This Study

\begin{tabular}{|c|c|c|c|c|}
\hline CBM & $\begin{array}{l}\text { Parent Protein } \\
\text { (Swiss-Prot Accession No.) }\end{array}$ & Organism & Specificity & Reference \\
\hline CtCBM3 & CipA (Q06851) & Clostridium thermocellum & Crystalline cellulose & $(29,30)$ \\
\hline SICBM20 & AmlA (O86876) & Streptomyces lividans & Starch & This study \\
\hline
\end{tabular}

of $C t$ CBM3-RFP and $C t$ CBM6-GFP. The character of the label in each case is very different-while CBM3 labels relatively broad regions of the cellulose crystals in a comparatively uniform manner, the interaction of CBM6 with the Cel7A-bound substrate results in a punctate labeling pattern (Figure 2D), possibly indicating single Cel7A molecules or small clusters of molecules. It is currently unclear whether the latter label is due to the interaction of CBM6 with Cel7A oligosaccharides, or whether the observed label reflects enzymatic action on the substrate that could produce or expose noncrystalline regions on the cellulose surface.

The primary cell wall is an example of a natural higher-order complex of carbohydrate-containing materials, which comprises networks of cellulose microfibrils embedded into colloidal matrices containing various branched polysaccharides (e.g., xyloglucans, arabinoxylans, and pectins), lignins, and glycoproteins $(4,40)$. To visualize the cellulose microfibrils that are as small as $5-10 \mathrm{~nm}$ in diameter, EM techniques and consequent extraction and dehydration processes are often required. However, these extensive sample preparation steps may alter the native molecular structure of cell walls. We thus employed correlative binding of the CBM3 and CBM6 probes to map cell wall structure. Parenchyma tissue was carefully cut from living $Z$. mays $\mathrm{L}$. stem, pulverized in liquid nitrogen, and small pieces of primary cell walls were then collected, washed, and suspended in distilled water prior to interaction with the CBM probes. Fluorescence microscopy imaging showed distinct binding patterns of $\mathrm{CtCBM3}$-GFP versus CtCBM6-RFP (Figure 2E). Notably, it appears that $C t$ CBM3-GFP binds to the fiber network, whereas $C t$ CBM6-RFP binds to the primary cell wall matrices, as shown clearly in Figure 2E. As noted in Table 1, the recognition specificity of $\mathrm{CtCBM} 3$ is for crystalline cellulose and that of $C t$ CBM6 for soluble polysaccharides or oligosaccharides. Using the CBM probes, we were therefore able to label and image directly plant cell wall cellulose macrofibrils at the molecular level of resolution. This approach supports our recently proposed model for the primary cell wall in which the macrofibrils, containing primarily crystalline cellulose, appear only on the uppermost surface (the plasma membrane side) of the dissected parenchyma cell wall (36).

In summary, we have successfully demonstrated the use of CBMs as molecular probes for mapping the chemistry and structure of various complex carbohydrate-containing materials, which then permits direct imaging of these macromolecules using fluorescence microscopy, TEM, and STEM correlatively at the molecular level of resolution. Numerous CBMs with finely tuned recognition specificities have been characterized; many are currently available but await characterization, and many more are undoubtedly yet to be discovered. An intriguing possibility yet to be explored is that the CBMs are essentially devices designed by nature that reflect the microheterogeneity believed to exist in most natural glycans. Thus, once fully characterized, these probes may be even more relevant as probes for examining natural carbohydrate-containing surfaces than antibodies elicited against distinct epitopes (8). Many of these CBMs are relatively stable molecules; therefore they are easily cloned and expressed in prokaryotic systems with GFP or other functional tags useful for further chemical and/or biological labeling studies (e.g., His-tag/QD labeling as demonstrated in this study). In addition,
CBMs are small polypeptides, usually containing $30-150$ amino acids. The size of most CBM proteins is about $3-4 \mathrm{~nm}$ in diameter, which is much more compact than most antibodies. This method thus simplifies the labeling procedure and provides higher spatial resolution than that provided by immunochemical and spectroscopic methods. The new methodology should be useful for applications in biomass characterization, pulp and paper, food and feed, biomedicine, biomaterials, and design of new hybrid bionanostructures $(35,36)$.

\section{ACKNOWLEDGMENTS}

The authors are grateful to $M$. Simon, B. Lin, and J. Wall (Brookhaven National Lab) for STEM studies (supported by USDOE OHER and NIH NIBIB EB 008121-23) and to $P$. Ahrenkiel (National Renewable Energy Laboratories) for TEM studies. This work was funded by the U.S. Department of Energy (DOE) Solar Photochemisry Program funded by OS/BES, Division of Chemical Sciences, Geosciences, and Biosciences under contract no. DE-AC36-99GO10337 and by the DOE Office of the Biomass Program. This research was also supported by the Israel Science Foundation (grant nos. 394/03 and 442/05) and by grants from the United States-Israel Binational Science Foundation (BSF), Jerusalem, Israel.

\section{COMPETING INTERESTS STATEMENT}

The authors declare no competing interests. 


\section{REFERENCES}

1. Lee, Y.C. and R.T. Lee. 1995. Carbohydrateprotein interactions: basis of glycobiology. Acc. Chem. Res. 28:321-327.

2. Taylor, M. and K. Drickamer. 2003. Introduction to Glycobiology. Oxford University Press, London.

3. Sharon, N. 2006. Protein-carbohydrate interactions - at the heart of biochemistry. The biochemist. 28:13-17.

4. Cosgrove, D.J. 2005. Growth of the plant cell wall. Nat. Rev. Mol. Cell Biol. 6:850-861.

5. Serpe, M.D., A.J. Muir, and A.M. Keidel. 2001. Localization of cell wall polysaccharides in nonarticulated laticifers of Asclepias speciosa. Torr. Protoplasma 216:215-226.

6. His, I., A. Driouich, F. Nicol, A. Jauneau, and H. Hofte. 2001. Altered pectin composition in primary cell walls of korrigan, a dwarf mutant of Arabidopsis deficient in a membrane-bound endo-1,4- $\beta$-glucanase. Planta 212:348-358

7. Guillemin, F., F. Guillon, E. Bonnin, M.F. Devaux, T. Chevalier, J.P. Knox, F. Liners, and J.F. Thibault. 2005. Distribution of pectic epitopes in cell walls of the sugar beet root. Planta 222:355-371.

8. McCartney, L., S.E. Marcus, and J.P. Knox. 2005. Monoclonal antibodies to plant cell wall xylans and arabinoxylans. J. Histochem. Cytochem. 53:543-546.

9. Berg, R.H., G.W. Erdos, M. Gritzali, and R.D. Brown. 1988. Enzyme-gold affinity labelling of cellulose. J. Electron Microsc. Tech. 8:371-379.

10. Blanchette, R.A., A.R. Abad, R.L. Farrell, and T.D. Leathers. 1989. Detection of lignin peroxidase and xylanase by immunocytochemical labeling in wood decayed by basidiomycetes. Appl. Environ. Microbiol. 55:1457-1465.

11. Reis, D., B. Vian, H. Chanzy, and J.C. Roland. 1991. Liquid crystal-type assembly of native cellulose-glucuronoxylans extracted from plant cell wall. Biol. Cell. 73:173-178.

12.Jung, H.G., F.R. Valdez, A.R. Abad, R.A. Blanchette, and R.D. Hatfield. 1992. Effect of white rot basidiomycetes on chemical composition and in vitro digestibility of oat straw and alfalfa stems. J. Anim. Sci. 70:19281935

13. Barrasa, J.M., A. Gutierrez, V. Escaso, F. Guillen, M.J. Martinez, and A.T. Martinez. 1998. Electron and fluorescence microscopy of extracellular glucan and aryl-alcohol oxidase during wheat-straw degradation by Pleurotus eryngii. Appl. Environ. Microbiol. 64:325-332.

14. Adams, E.L., P.A. Kroon, G. Williamson, H.J. Gilbert, and V.J. Morris. 2004 Inactivated enzymes as probes of the structure of arabinoxylans as observed by atomic force microscopy. Carbohydr. Res. 339:579-590.

15. Benhamou， N., N. Gilboa-Garber, J. Trudel, and A. Asselin. 1988. A new lectin-gold complex for ultrastructural localization of galacturonic acids. J. Histochem. Cytochem. 36:1403-1411.

16. Boraston, A.B., D.N. Bolam, H.J. Gilbert, and G.J. Davies. 2004. Carbohydrate-binding modules: fine-tuning polysaccharide recognition. Biochem. J. 382:769-781.

17. Carrard, G., A. Koivula, H. Soderlund, and P. Béguin. 2000. Cellulose-binding domains promote hydrolysis of different sites on crystalline cellulose. Proc. Natl. Acad. Sci. USA 97:10342-10347.
18. McLean, B.W., A.B. Boraston, D. Brouwer, N. Sanaie, C.A. Fyfe, R.A. Warren, D.G. Kilburn, and C.A. Haynes. 2002. Carbohydrate-binding modules recognize fine substructures of cellulose. J. Biol. Chem. 277:50245-50254

19. Hilden, L. and G. Johansson. 2004. Recent developments on cellulases and carbohydrate-binding modules with cellulose affinity. Biotechnol. Lett. 26:1683-1693.

20. Hilden, L., G. Daniel, and G. Johansson. 2003. Use of a fluorescence labelled, carbohydrate-binding module from Phanerochaete chrysosporium Cel7D for studying wood cell wall ultrastructure. Biotechnol. Lett. 25:553558

21. McCartney, L., H.J. Gilbert, D.N. Bolam, A.B. Boraston, and J.P. Knox. 2004. Glycoside hydrolase carbohydrate-binding modules as molecular probes for the analysis of plant cell wall polymers. Anal. Biochem. 326:49-54.

22. McCartney, L., A.W. Blake, J. Flint, D.N. Bolam, A.B. Boraston, H.J. Gilbert, and J.P. Knox. 2006. Differential recognition of plant cell walls by microbial xylan-specific carbohydrate-binding modules. Proc. Natl. Acad. Sci. USA 103:4765-4770.

23. Lehtio, J., J. Sugiyama, M. Gustavsson, L. Fransson, M. Linder, and T.T. Teeri. 2003. The binding specificity and affinity determinants of family 1 and family 3 cellulose binding modules. Proc. Natl. Acad. Sci. USA 100:484-489.

24. Sugiyama, J., R. Vuong, and H. Chanzy. 1991. Electron diffraction study of the two crystalline phases occurring in native cellulose from an algal cell wall. Macromolecules 24:4168-4175.

25. Imai, T., J.L. Putaux, and J. Sugiyama. 2003. Geometric phase analysis of lattice images from algal cellulose microfibrils. Polym. 44:1871-1879.

26. Vlasenko, E.Y., H. Ding, J.M. Labavitch, and S.P. Shoemaker. 1997. Enzymatic hydrolysis of pretreated rice straw. Bioresour. Technol. 59:109-119.

27. Ding, S.Y., M. Jones, M.P. Tucker, J.M. Nedeljkovic, J. Wall, M.N. Simon, G. Rumbles, and M.E. Himmel. 2003. Quantum dot molecules assembled with genetically engineered proteins. Nano Lett. 3:1581-1585.

28. Wall, J.S., M.N. Simon, and J.F. Hainfeld. 1997. The BNL STEM facility, pp. 277278. In G.W. Bailey (Ed.), Microscopy and Microanalysis. Springer, Cleveland.

29. Tormo, J., R. Lamed, A.J. Chirino, E. Morag, E.A. Bayer, Y. Shoham, and T.A. Steitz. 1996. Crystal structure of a bacterial family-III cellulose-binding domain: a general mechanism for attachment to cellulose. EMBO J. 15:5739-5751.

30. Morag, E., A. Lapidot, D. Govorko, R. Lamed, M. Wilchek, E.A. Bayer, and Y. Shoham. 1995. Expression, purification and characterization of the cellulose-binding domain of the scaffoldin subunit from the cellulosome of Clostridium thermocellum. Appl. Environ. Microbiol. 61:1980-1986.

31. Czjzek, M., D.N. Bolam, A. Mosbah, J. Allouch, C.M. Fontes, L.M. Ferreira, O. Bornet, V. Zamboni, et al. 2001. The location of the ligand-binding site of carbohydrate-binding modules that have evolved from a common sequence is not conserved. J. Biol. Chem. 276:48580-48587.

32. Henshaw, J.L., D.N. Bolam, V.M. Pires, M. Czjzek, B. Henrissat, L.M. Ferreira, C.M. Fontes, and H.J. Gilbert. 2004. The family 6 carbohydrate binding module CmCBM6-2 contains two ligand-binding sites with distinct specificities. J. Biol. Chem. 279:21552 21559.

33.Lammerts van Bueren, A.L., C. Morland, H.J. Gilbert, and A.B. Boraston. 2005 . Family 6 carbohydrate binding modules recognize the non-reducing end of $\beta$-1,3-linked glucans by presenting a unique ligand binding surface. J. Biol. Chem. 280:530-537.

34. Atalla, R.H. and D.L. VanderHart. 1984 Native cellulose: a composite of two distinct crystalline forms. Science 223:283-285.

35. Ding, S.-Y., S. Smith, Q. Xu, J. Sugiyama, M. Jones, G. Rumbles, E.A. Bayer, and M.E. Himmel. 2005. Towards building ordered arrays of quantum dots using cellulosomal proteins. Indust. Biotechnol. 1:198206.

36. Ding, S.Y. and M.E. Himmel. 2006. The maize primary cell wall microfibril: a new model derived from direct visualization. J. Agric. Food Chem. 54:597-606.

37. Wood, T.M. 1988. Preparation of crystalline, amorphous and dyed cellulase substrates. Methods Enzymol. 160:19-25

38. Ding, S.Y., G. Rumbles, M. Jones, M.P. Tucker, J.M. Nedeljkovic, M.N Simon, J. Wall, and M.E. Himmel. 2004. Bioconjugation of (CdSe)ZnS quantum dots using a genetically engineered multiple polyhistidine tagged cohesin/dockerin protein polymer. Marcromol. Mater. Eng. 289:622 628.

39. Vlasenko, E.Y., A.I. Ryan, C.F. Shoemaker, and S.P. Shoemaker. 1998. The use of capillary viscometry, reducing end-group analysis, and size exclusion chromatography combined with multi-angle laser light scattering to characterize endo-1,4- $\beta$-D-glucanases on carboxymethylcellulose: a comparative evaluation of three methods. Enzyme Microb. Technol. 23:350-359.

40. Somerville, C., S. Bauer, G. Brininstool, M. Facette, T. Hamann, J. Milne, E. Osborne, A. Paredez, et al. 2004. Toward a systems approach to understanding plant cell walls. Science 306:2206-2211.

41. Levy, L. 2001. MSc. Theis: Characterization of a family 6 carbohydrate binding module $(\mathrm{CBM})$ and its comparison with other CBM-tags for phage display, p. 122. In Department of Molecular Microbiology and Biotechnology. Tel Aviv University, Rama Aviv.

Received 14 March 2006; accepted 30 June 2006.

Address correspondence to Shi-You Ding, Energy Sciences Directorate, National Renewable Energy Laboratory, Golden, CO 80401, USA.e-mail: shi_you_ding@nrel. gov or Edward A. Bayer, Department of Biological Chemistry, Weizmann Institute of Science, Rehovot, 76100, Israel. e-mail: ed.bayer@weizmann.ac.il

To purchase reprints of this article, contact: Reprints@BioTechniques.com 